\title{
A Decade of Capacitated Arc Routing
}

\author{
Sanne Wøhlk \\ Department of Business Studies \\ Aarhus School of Business, Aarhus University \\ Denmark \\ sanw@asb.dk
}

Summary. Arc Routing is the arc counterpart to node routing in the sense that focus regarding service and resource constraints are on the arcs and not on the nodes. The key problem within this area is the Capacitated Arc Routing Problem (CARP), which is the arc routing counterpart to the vehicle routing problem. During the last decade, arc routing has been a relatively active research area with respect to lower bounding procedures, solution approaches and modeling. Furthermore, several interesting variations of the problem have been studied. We survey the latest research within the area of arc routing focusing mainly on the CARP and its variants.

Key words: Arc routing; CARP; recent research.

\section{Introduction}

The Capacitated Arc Routing Problem is the problem of servicing a set of streets in a street network using a fleet of capacity constrained vehicles initially located at a central depot. The objective of the problem is to minimize the total routing cost. Theoretically, the CARP is an arc routing counterpart to the Vehicle Routing Problem and has been proved to be $\mathcal{N} \mathcal{P}$-hard.

In practice, the CARP and its variants occur in many aspects of both public and private businesses, where street segments rather than specific points need service. Often several extra constraints must be taken into account, examples of which are a heterogeneous fleet of vehicles, service time restrictions, prohibited U-turns, and one-way streets. For details, we refer the reader to Assad and Golden, [10], and Dror, [37].

Two of the earliest real life problems studied in an arc routing setting are the Street Sweeping problem and the Electric Meter Reading problem. The problem of Refuse Collection can be modeled as a CARP where the goal is to spread the load evenly among the tours. Various aspects of Refuse Collection have been considered recently in $[3,4,8,14,55,60,81]$.

The problem of spreading salt or sand on streets for the purpose of ice control is called the Winter Gritting problem. This problem can be modeled 
as a CARP with multiple depots, [29], and in some cases may include time windows or other complicating constraints. For a recent review of Winter Gritting and Snow Removal problems, the reader is referred to [27, 28]. The arc routing problem of monitoring a road network is considered in [78]. The problem of planning the monitoring is modeled as a directed CARP, where the authors consider the problem of re-planning when tours are not completed due to unexpected events.

During the last decade, the CARP and its variants have been extensively studied. In this chapter we offer a survey of this research. Even though the main emphasis will be on the recent research, earlier work will be included whenever this is appropriate for completeness. We hope that this will help researchers to quickly obtain an overview of the problem and to guide them to explore new and untested aspects of the field of research.

The remainder of this chapter is organized as follows: In Section 2, we give a historical overview of arc routing and formally define the most important arc routing problems. We also state some complexity results and point out the relation among various problems. In Section 3, we consider the classical CARP with respect to heuristics, lower bounds, and exact optimization. Section 4 covers different variants and extensions of the CARP, all of which can be justified in real life applications of the problem. These include a multi-depot version of the problem, alternative objective functions, and the inclusion of time windows. Finally, in Section 5, we offer our directions for future research within the area of capacitated arc routing.

\section{A Historical Perspective and Problem Definitions}

The study of arc routing problems began on August 26, 1735 when Leonhard Euler presented his solution to the Königsberg bridge problem, [86]. Theoretically, the problem, now known as the Euler Tour Problem, is as follows. Given a connected graph $G=(N, E)$ find a closed tour that visits every edge in E exactly once, or determine that no such tour exists. Euler proved that an Euler Tour exists if and only if every node in $G$ has even degree and many years later Fleury presented an algorithm for constructing an Euler Tour [51].

The next arc routing problem to be studied was the Chinese Postman Problem (CPP) first suggested by the Chinese mathematician Kwan Mei-Ko in 1962, [82]. The problem is formally stated as follows: Given a connected graph $G=(N, E, C)$, where $C$ is a distance matrix, find a tour which passes through every edge at least once and does this in the shortest possible way. When $G$ is completely directed or completely undirected, the CPP can be solved in polynomial time, $[39,31]$, but when $G$ is a mixed graph, the problem becomes $\mathcal{N} \mathcal{P}$-hard, [85]. Many variants of the problem have been studied, including the Windy Postman Problem, [83], and the Hierarchical Postman Problem, [38]. For a survey on the Chinese Postman Problem and some of its variants we recommend [44]. 
In 1974, Orloff suggested the Rural Postman Problem (RPP), [84], which is formally stated as follows: Given an undirected graph $G=(N, E, C)$, where $C$ is the cost matrix for the edges, find a minimum cost tour, which passes through every edge in a subset $R \subseteq E$ at least once. The RPP is $\mathcal{N P}$-hard, [68], where the hardness comes from determining how the tour should connect the various components of edges in $R$. It can be shown that the class of RPP and the class of Traveling Salesman Problems are equivalent. A 3/2-approximation algorithm for the RPP is given in [67] where it is also noted that the problem can be solved to optimality in polynomial time if the graph spanned by the set $R$ consists of only a fixed number of components. Several heuristics and methods for solving the problem to optimality have been presented in the literature, see [61] for a recent update. Many variants of the problem have been considered, e.g. the Rural Postman Problem with Deadline Classes, [41], in which the set $R$ of required edges are partitioned into several sets, which in turn are ordered so that all the edges in an earlier set must be traversed before any of the edges in a later set are traversed. For a survey on the RPP we recommend [45].

The Min-Max $k$-Chinese Postman Problem (MM $k$-CPP), was suggested by Frederickson et al. in 1978, [53], and is formally stated as follows: Given a connected undirected graph $G=(N, E, C)$, where $C$ is a distance matrix, with a special depot node, find $k$ tours, starting and ending in the depot node, such that every edge is covered by at least one tour and the length of the longest tour is minimized. It should be noted that for this problem the objective is to minimize the makespan, whereas most other problems with multiple postmen seek to minimize the total distance traveled. A $2-1 / k$-approximation algorithm is given for the MM $k$-CPP in [53].

The Capacitated Arc Routing Problem, which was first suggested by Golden and Wong in 1981, [58], is formally stated as follows: Given a connected undirected graph $G=(N, E, C, Q)$, where $C$ is a cost matrix and $Q$ is a demand matrix, and given a number of identical vehicles each with capacity $W$, find a number of tours such that 1) Each arc with positive demand is serviced by exactly one vehicle, 2) The sum of demand of those arcs serviced by each vehicle does not exceed $W$, and 3) The total cost of the tours is minimized. The Capacitated Chinese Postman Problem (CCPP), which is a variation of the CARP where every edge in the graph has a strictly positive demand was first suggested by Christofides in 1973, [31]. Both the CARP and the CCPP are $\mathcal{N} \mathcal{P}$-hard, [58], and it can be proved that even obtaining a $3 / 2$-approximation of either of the two problems is $\mathcal{N} \mathcal{P}$-hard, [58].

It can be shown that the Vehicle Routing Problem (VRP) can be transformed into the CARP, [58], and that the CARP can be transformed into the VRP, [11, 13, 35], making the two classes of problems equivalent. For all three transformations of the CARP into the VRP, the resulting VRP instance requires either fixing of variables or the use of edges with infinite cost. Moreover, the resulting VRP graph is a complete graph of larger size. Therefore 
the problem size increases and the planar structure of a usual CARP graph is lost.

Somewhere between node routing and arc routing we find the so-called Stringed VRP, in which customers are to be serviced as in the VRP, but some of these customers are located along the streets as in the refuse collecting problem. From an arc routing point of view these customers would be considered as demand of an arc and must be serviced together, but in the Stringed VRP they need not be serviced by the same vehicle. The Stringed VRP has been considered by [80], where the authors use aggregation of these special customers and solve the problem to near optimality using a Tabu Search algorithm.

\section{The Classical CARP}

In this section, we consider the Capacitated Arc Routing Problem in the classical setup as defined in the previous section. In Section 3.1, we consider heuristics suggested for solving the CARP during the last decade. This includes a few problem specific heuristics and numerous metaheuristic approaches. A survey of the various lower bounding procedures presented for the CARP is given in Section 3.2. Finally, an overview of exact solution approaches used to solve the CARP is given in Section 3.3. This order of presentation is motivated by the historical development, which started with simple problem specific heuristics, whereas methods for solving the problem exactly were suggested much later.

Four sets of benchmark test instances are used for computational experiments for the CARP. These are usually referred to as the Gdb, [12], Val, [22], Kshs, [69], and Eglese, [43] instances, and can be downloaded from [16].

\subsection{Heuristics for the CARP}

During the 1980s, problem specific heuristics were the common method for solving the CARP. These classical algorithms include the Construct-Strike algorithm, the Path-Scanning method, and the Augment-Merge algorithm. For a survey on these classical algorithms we refer the reader to [37, 92]. The performance of the classical problem specific heuristics are generally 10 to 40 percent above the optimal solution.

More recently other problem specific heuristics have been proposed. These include the Double Outer Scan heuristic, [92], which combines the AugmentMerge algorithm and the Path Scanning method, and the Node Duplication heuristic, [92], which uses ideas similar to those in the Node Duplication Lower Bound, [65]. The former is illustrated in Figure 1, where the idea in the construction of one tour is shown. In the latter, a Node Duplicated network is constructed and the edges of a minimum cost perfect matching are added to the demand edges. These edges together now form an Euler tour. Methods for partitioning this tour into feasible vehicle tours include simple forward and 


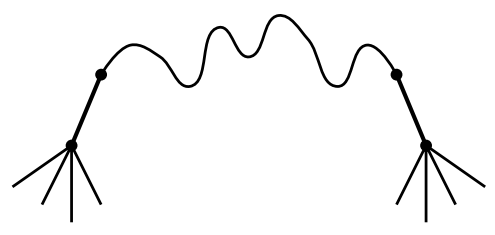

Depot

Fig. 1. Illustration of the Double Outer Scan heuristic.

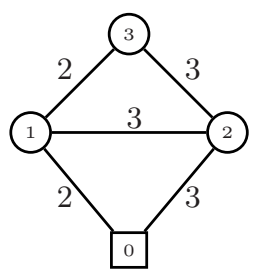

A: Original graph

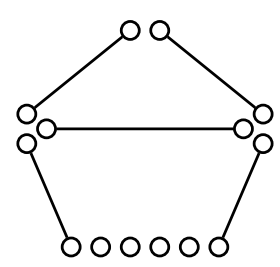

B: Node Duplicated Network

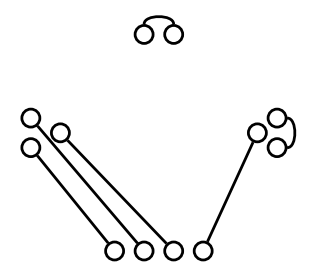

C: Matching

Fig. 2. Illustration of the Node Duplication heuristic.

optimal partitioning. This algorithm is illustrated in Figure 2, where A shows the original graph with numbers on the edges indicating the cost. Node 0 is the depot node, the five edges have unit demand, and the vehicle capacity is 2. B shows the node duplicated network. The total cost of the demand edges is 13. Finally, $\mathrm{C}$ gives a minimum cost perfect matching of cost 9 . Combining

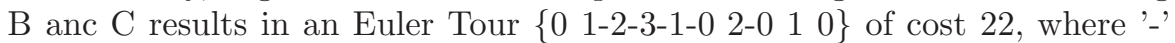
indicates service. This tour is partitioned into three vehicle tours, $\left\{\begin{array}{l}01-2-3 \\ 1\end{array}\right.$ $0\},\left\{\begin{array}{lll}0 & 1 & 3-1-0\end{array}\right.$, and $\left\{\begin{array}{ll}0 & 2-0\end{array}\right\}$, with total cost $12+8+6=26$.

The A-ALG algorithm by Wøhlk, [92], is an $\frac{7}{2}-\frac{3}{W}$-approximation algorithm for the CARP, where $W$ is the vehicle capacity. The idea is to use the $\frac{3}{2}$-approximation algorithm for the RPP by Frederickson, [52], to construct a giant tour, which is partitioned into vehicle tours using optimal partitioning. Both the Node Duplicated Heuristic and the A-ALG are highly competitive to the classical problem specific heuristics.

During the last decade, most advances in the development of heuristics for the CARP have dealt with metaheuristics. For a general description of the various metaheuristics we refer the reader to [57].

Eglese, [40], considers a winter gritting problem, which is modeled as a CARP with extra complicating constraints specific to the case studied. He solves this problem using a Simulated Annealing algorithm. Wøhlk, [92], suggests a Simulated Annealing algorithm for the classical CARP, where the order 
of the edges on a giant tour is changed during the algorithm, and at each step the optimal partitioning of the tour is calculated.

Several Tabu Search algorithms have been constructed for the CARP. The first Tabu Search algorithm called CARPET, [62], was suggested by Hertz et al. in 2000. Here infeasible solutions are allowed but penalized in the objective function. This algorithm outperformed the existing algorithms at that time and is still one of the best performing algorithms for the CARP. For the Multi Depot version of the CARP, a Tabu Search algorithm, has been suggested by Amberg et al., [6], in 2000. In 2003 Greistorfer, [59], combined Tabu Search with Scatter Search to construct a Tabu Scatter Search for the CARP. Finally a completely deterministic Tabu Search algorithm has recently been suggested by Brandâo and Eglese, [26], which, with varying extent, penalizes infeasible solutions in the objective function and alternates between different neighborhood structures.

Lacomme et al. presented a Genetic Algorithm in 2001, [71], and a Memetic Algorithm in 2004, [74]. In both algorithms crossover is performed on a giant tour, and fitness of a chromosome is based on the partitioning of the tour into vehicle tours. Currently these algorithms are among the very best performing for the CARP. Chu et al., [34], presented a Scatter Search algorithm for a periodic version of the CARP. When tested on instances of the classical CARP their algorithm is competitive to CARPET, but with longer computation times.

One of the younger generations of metaheuristics is that of an Ant Colony System. Lacomme et al., [77], propose such an algorithm where two types of ants are used, elitist ants which make the solution converge towards a minimum cost solution and non-elitist ants which ensure diversification to avoid getting trapped in a local minimum. This algorithm works on a graph where edges are replaced by two directed arcs. The authors report results competitive to the best algorithms with respect to solution quality but with longer computation times. Doerner et al., [36], applied an Ant Colony System to the CARP where they worked directly on the undirected graph. The authors report limited success.

A Guided Local Search algorithm has been presented for the CARP by Beullens et al., [23], in 2003, where the distance of each edge is penalized according to some function which is adjusted throughout the algorithm. Computational experiments show that this approach is promising.

A Variable Neighborhood Descent algorithm has been presented by Hertz and Mittaz, [64]. They suggest the first neighborhood to be based on the procedures ADD and DROP, whereas the remaining neighborhoods are based on merging a number of tours succeeded by a sequence of SWITCH steps and completed by CUT and SHORTEN, all of which are well-known procedures originally suggested by Hertz et al., [63]. The reported results are among the best to date. 


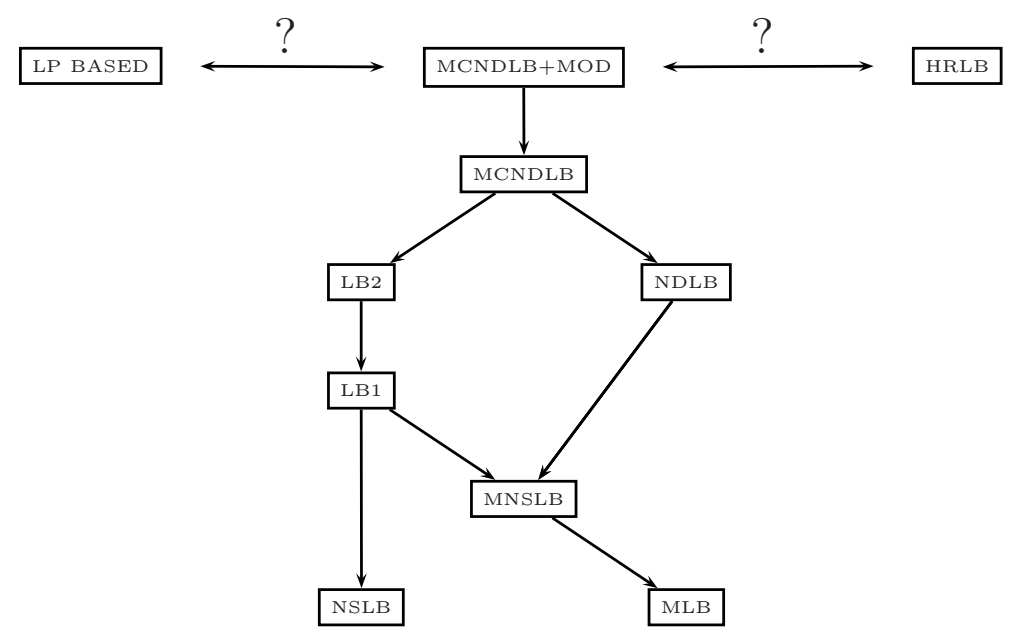

Fig. 3. Relationships among lower bounds for the CARP.

\subsection{Lower Bounds for the CARP}

There is a tradition for using combinatorial lower bounds based on minimum cost perfect matchings for the CARP. In Figure 3, the relationship is shown among the various lower bounds for the CARP. Here an arrow from bound $x$ to $y$ indicates that bound $x$ outperforms bound $y$, i.e., it has been proved that $x(\sigma) \geq y(\sigma)$ for any instance $\sigma$ of the problem. It should be noted that the Hierarchical Relaxations Lower Bound (HRLB) has only been experimentally compared with the other bounds, so even though it performs well on the instances tested, to our knowledge, it has not been proved to outperform any of the other bounds for all instances, which explains its position in the figure.

The first lower bound to be proposed for the CARP is the Matching Lower Bound (MLB) in 1981, [58]. Next, the Node Scanning Lower Bound (NSLB) was suggested in 1987. It is based on logic arguments that bound the length of the path the vehicles must traverse in the beginning and end of its tour. Combining the bounds gives us the Matching - Node Scanning Lower Bound (MNSLB). In 1992 an improvement of the MLB, called the LB1, was suggested. The Node Duplication Lower Bound (NDLB) from 1992 is based on a matching in a network where the nodes are duplicated and connected by the shortest paths if the combination of the corresponding demand edges is possible in a legal vehicle tour.

The five lower bounds, MLB, NSLB, MNSLB, NDLB, and LB1 all estimate the number of vehicles needed to service the graph based on the cut $(\{1\}, G \backslash$ $\{1\})$, where node 1 is the depot. In [90] it is suggested to consider not only one cut as in the previous bounds, but a whole family of disjoint cuts. This method increases the complexity of the algorithms but gives stronger results. 
The next couple of lower bounds for the CARP are based on that idea. Let $U$ be a set of nodes that includes the depot and let $G(U)$ be the graph induced by $U$. For each set of cuts, $(U, \bar{U})$ a lower bound on the cost of the edges in $G(U)$ and an estimate of the cost of the edges in $G \backslash G(U)$ is calculated. In [90], estimates of the cost of the edges in $G(U)$ is made by a construction similar to the one used in MLB. The LB2 algorithm improves this aspect by estimating the cost using the LB1 algorithm.

For instances of the CARP where the number of vehicles used is fixed, the two lower bounds, LB3 and LB4 have been suggested. For description of the above mentioned lower bounds we refer the reader to [1, 92], where references are also given to the original papers.

The Hierarchical Relaxation Lower Bound (HRLB), presented by Amberg and Voß in 2002, [7], is an iterative bound like LB2 but here the cuts that are iterated over are not disjoint. HRLB starts out by solving the CPP relaxation of the CARP. In each iteration more constraints are added to the problem, the cut set is extended and the relaxation is solved again. No relationships have been proved between HRLB and the other bounds, but in practice HRLB has shown to perform very well.

The Multiple Cuts Node Duplication Lower Bound (MCNDLB), suggested by Wøhlk in 2006, [93], uses the same disjoint cuts strategy as LB2, but at each iteration a stronger matching network, which is similar to the one used in the NDLB, is used to estimate the cost of servicing the edges in $G \backslash G(U)$. MCNDLB was proved to be stronger than both LB2 and NDLB. In [1], Ahr improved the MCNDLB by considering more cuts than the successive disjoint ones considered in LB2 and MCNDLB. This is done by adding the nodes to be added to $U$ in each iteration one by one, while calculating the cost of a matching in the new $G \backslash G(U)$ for each of these nodes. The algorithm with this modification is called MCNDLB+MOD. Computational experiments show that for some instances these extra calculations result in better bounds.

\subsection{Exact Methods for the CARP}

Since the mathematical formulation of the CARP by Golden and Wong in 1981, [58], several different formulations have been proposed for the problem, ranging from dense, to sparse to supersparse. We refer the interested reader to [42] for details on these formulations and to [21] for an overview of valid inequalities and separation routines.

The first attempt to solve the CARP exactly was by Hirabayashi et al., [66], in 1992 by the use of Branch-and-Bound, where the Node Duplication Lower Bound was used to calculate lower bounds for the subproblems and branching was performed on a single edge of the node duplicated network. Using this algorithm, the authors are able to solve a set of CARP instances with from 15 to 50 demand edges to optimality.

In [19], Belenguer and Benavent present a Cutting Plane algorithm for the CARP, which is partly based on several classes of valid inequalities presented 
earlier by the same authors, [18]. Using their algorithm, the authors are able to reach the best existing lower bound for all test instances, and can improve the existing lower bounds for several instances. They reduce the average gap between upper and lower bounds to less than one percent for the Gdb, Kshs, and Val instances and less than 2.4 percent for the Eglese instances.

Both Baldacci and Maniezzo, [13], and Aragão et al., [35], propose an exact solution strategy for the CARP which is based on transforming the problem to a corresponding node routing problem (VRP), which is in turn solved by state-of-the-art algorithms. The transformation used in these papers is very similar to the one used in the network construction for the node duplicated lower bound, except for duplication of the depot. In both papers, the resulting VRP requires fixing a set of edges to belong to the solution set. Both papers report computational results that are highly competitive to the existing ones.

A Branch-Price-and-Cut algorithm is suggested for the CARP by Belenguer et al. in [20]. Only the average performance of the algorithm is given and compared with the Cutting Plane algorithm given in [19]. With the Branch-Price-and-Cut algorithm the average lower bound is $0.07,0.39$, and 2.36 percent below the best known solution for the Gdb, the Val, and the Eglese instances, respectively. These results are better than the average lower bounds obtained in [19], but not as good as the ones obtained with the method presented in [35]. No comparison of the running time is reported.

Exact solution using a Branch-Price-and-Cut algorithm is also considered by Letchford and Oukil, [79]. Their goal is to use the fact that CARP networks are very sparse since they represent street networks. A column generation approach is used, and the pricing problem is considered in two versions. The first one allows non-elementary tours and is solved by a dynamic programming type of algorithm. The second one only allows elementary tours, which is an $\mathcal{N} \mathcal{P}$-hard problem, and is solved with a Cut-and-Branch algorithm. The algorithms are currently being tested and compared to existing work.

When using column generation to solve the CARP, the subproblem becomes the problem of finding a tour which starts and ends at the depot, does not exceed the vehicle capacity and minimizes the total cost in a graph where some demand edges have negative cost since the cost in this graph corresponds to the reduced cost of the edge. Ignoring the capacity constraint and inverting the cost structure, this problem is the Privatized Rural Postman Problem (PRPP), [9], which could also be called the Prize Collecting Arc Routing Problem, and is formally stated as follows: Given an undirected graph $G=(N, E)$, with a special depot node, $d$. Let $c_{e}$ be the cost of traversing the edge $e$ and let $b_{e}$ be the profit obtained the first time the edge $e$ is traversed. Let $t_{e}$ be an integer indicating the number of traversals of the edge e. The goal is to find a single tour, $T$, starting and ending in $d$, which maximizes $\sum_{e \in T}\left(b_{e}-t_{e} c_{e}\right)$, i.e., which maximizes the total profit.

This problem can intuitively be considered as an arc version of the Prize Collecting Traveling Salesman Problem, which is a node routing problem. To illustrate the problem, imagine a computer game where the goal is to traverse 
a street network with the purpose of collecting treasures, maybe even in a stochastic setup. In [9], Aráoz et al. give several mathematical models for the PRPP, prove dominance relations among these, and deal with preprocessing aspects of their final model. Furthermore, they give polynomial time algorithms for the PRPP on a tree and a cactus, i.e., a graph where each edge is contained in at most one cycle.

\section{Variations of the CARP}

In this section, we consider variations of the classical CARP. Each of the variations considered reflects situations occurring in real life applications. In Section 4.1, we consider CARP defined on directed or mixed graphs, whereas in Section 4.2, we deal with alternative objective functions including Min-Max $k$-CPP, which is a CARP like problem with several vehicles but excluding capacity constraints. We consider the problem of including time window constraints in Section 4.3. Sections 4.4 and 4.5 deal with CARP with multiple depots and with mobile depots, respectively. A version of the problem, where not all vehicles are able to service all edges is considered in Section 4.6. The periodic CARP is considered in Section 4.7, and finally Section 4.8 considers a stochastic version of the problem.

\subsection{CARP on Directed or Mixed Graphs}

The classical CARP is defined on an undirected graph but several real life applications of the problem must take into account the existence of one-waystreets and streets where the two sides must be serviced in parallel. This requires the definition of the CARP on a directed and mixed graph respectively. These variations of the problem are referred to as DCARP and MCARP. Benchmark instances for the MCARP are available in [17].

The mixed CARP is considered extensively by Belenguer et al. in [15], where three problem specific heuristics, Augment-Merge, Path-Scanning, and Ulusoy's heuristic, are improved and changed to fit the problem and to include extra complications such as windy edges, prohibited turns, and several dumping sites. Furthermore, the Memetic Algorithm by Lacomme et al., [74], is adapted to the MCARP. Finally, the authors give a supersparse LP formulation of the MCARP, which is used in a Cutting Plane algorithm to obtain strong lower bounds for the problem. Computational experiments show that the gap between the lower bound and their Memetic algorithm is less than one percent for the test instances in [17].

A directed version of the CARP has been considered by Welz, [89], in order to derive optimal solutions. The author presents valid inequalities and separation algorithms for an ILP formulation of this problem. Lacomme et al., [72], consider an extension of the CARP, where mixed graphs, prohibited turns, and non-trivial cost structures are included. A mathematical model that includes these considerations is suggested for this problem. 


\subsection{CARP with Alternative Objective Functions}

The usual objective in routing problems is to minimize the total distance traversed. But in several real life applications other objectives are just as important. This could be minimizing the total number of vehicles used, equalizing the load of the tours, or minimizing the length of the longest tour.

In [88], Ulusoy considers a version of the CARP where a vehicle includes a fixed cost if it is used and where the vehicles differ in capacity. Therefore, the objective function is to minimize the total travel cost plus the total fixed cost incurred by the use of vehicles. Both the case with an unlimited number of each vehicle type and the case where the number of each vehicle type is bounded are considered. A heuristic is presented which first constructs a giant tour and then splits the tour by solving a Shortest Path Problem which takes vehicle capacities and costs into account.

Lacomme et al., [75, 76], consider the Multi Objective CARP defined as the classical CARP where the objective is not only to minimize the total routing cost, but also to minimize the makespan, i.e., the length of the longest tour. With this objective the problem can be viewed as a mix between the CARP and the Min-Max K-Chinese Postman Problem, [53]. The authors present a generic algorithm for solving the Multi Objective CARP.

The Min-Max $k$-Chinese Postman Problem, [53], can be considered as a CARP where the vehicle capacity is infinite and the goal is to minimize the length of the longest tour. The problem is extensively studied by Ahr in [1]. The first heuristic for the MM $k$-CPP, which is presented by Frederickson et al. in [53], is based on constructing a giant tour which is subsequently partitioned into $k$ tours of roughly equal length. In [1], Ahr presents a heuristic based on the Augment-Merge algorithm for the CARP along with a new algorithm based on the cluster first - route second idea. A Tabu Search algorithm is presented for the MM $k$-CPP by Ahr and Reinelt in [2]. This algorithm is tested on a huge set of test instances with up to 392 edges. Computational testing shows that the results obtained by this algorithm is up to 30 percent better than the ones obtained with the construction heuristics and reduces the gap to the best lower bound to less than 10 percent (20 percent for a few instances). Two simple lower bounds are given directly for the MM $k$ CPP by Frederickson et al. in [53]. In [1], many of the combinatorial lower bounds originally presented for the CARP are adapted to work for the MM $k$-CPP using a modified notion of forbidden edges and the required number of postmen needed for a node set. The same text presents a Branch-and-Cut algorithm for the MM $k$-CPP using a new set of valid inequalities, which improved the lower bound obtained by about 5 percent on average.

A version of the directed CARP where the cost incurred by each arc depends on the time of service is considered by Gendreau et al. in [54] and is referred to as the CARP with Time-Dependent Service Costs. For real world problems such as winter gritting it can be argued that the CARP with this type of cost structure is more realistic than imposing hard time windows. In 
[54] the problem is solved to optimality by converting it to an equivalent node routing problem with Time-Dependent Service Costs. Since the problem is directed, this transformation can be performed without a large increase in the problem size. The resulting node routing problem is solved by column generation, where a labeling algorithm is used to generate columns.

\subsection{CARP with Time Windows}

The CARP with Time Windows (CARPTW) is defined as the classical CARP with the extra requirement that the service of each demand edge must begin within some pre-specified time window. Benchmark instances for the CARPTW can be downloaded from [91].

CARPTW occurs in some of the applications of arc routing. Flight legs in Airline Scheduling have a fixed departure time and can therefore be considered as having a time window of zero length or a very short time window if some flexibility is allowed. Street Sweeping, [25], has restrictions with respect to the time during which the sweeping may be performed, and routing of winter gritters, [40], where some streets must be serviced within two hours, others within four etc. can be considered as CARPTW where the time windows are rather wide.

Various aspects of the CARPTW are considered by Wøhlk in [92]. Two mathematical models are given for the problem, one based on constructing a node duplicated network on which the ILP model is built and one based on a transformation to the equivalent node routing problem, the VRPTW. Wøhlk shows how to improve the lower bound, MCNDLB for the classical CARP, [93], when used for the CARPTW.

In [92], a version of the Path-Scanning algorithm which chooses edges based on their time windows is presented and a new heuristic, the Preferable Neighbor heuristic is suggested. This algorithm is based on constructing a set of feasible vehicle tours that looks promising due to some pre-specified criteria, and the set covering problem defined by these tours is then solved to optimality. Computational testing indicates that the results obtained with this algorithm are on average 1.2 percent above the lower bound for the instances given in [91]. A Greedy Randomized Adaptive Search Procedure (GRASP) with Path Relinking is suggested for the CARPTW by Labadi et al. in [70]. The algorithm is based on a Randomized Path-Scanning heuristic and a new heuristic based on a route first - cluster second idea. Local search is used to improve each solution found using OR-OPT, SWAP, and 2-OPT, and Path Relinking is used to lead a solution towards structures that seem favorable. Computational results show that this algorithm obtains results that are on average 0.8 percent above the lower bound.

\subsection{Multi Depot CARP}

The Multi Depot CARP (MD-CARP) is defined as the classical CARP, where each vehicle is located in one of several depots from which it must start and 
end its tour. The most common variation of MD-CARP is where each vehicle must return to the depot from which it originated, but one could also consider the case where each vehicle just has to return to some depot independently of its starting location. Often a heterogeneous fleet of vehicles is considered in the MD-CARP. The MD-CARP frequently occurs in practice in mail delivery, winter gritting, and refuse collection. Some theoretical aspects of the MDCARP are considered by Wøhlk in [92], whereas Cattrysse et al., [29, 30], consider the long term planning problem of dividing the area into districts with one depot.

The problem of assigning streets to depots and constructing vehicle tours simultaneously is considered by Amberg et al. in [6]. Their solution strategy is quite unique for arc routing as the authors, after constructing a giant tour, transform the problem into an Arc-Constrained Capacitated Minimum Spanning Tree Problem (CMST). This problem is then solved heuristically, and the solution is improved by Local Search. Finally, a MD-CARP solution is derived from the CMST solution, and the resulting tours are improved by a simple route optimization procedure.

Ghiani et al., [56], consider a variation of the MD-CARP, referred to as the Capacitated Arc Routing Problem with Intermediate Facilities (CARP-IF). The problem is defined as the CARP with one depot, but has a set of nodes known as intermediate facilities, IF. The vehicles start and end at the depot but they can recharge their capacity in any of the intermediate facilities. For practical purposes the IFs can be dump sites for refuse or storage halls for salt for winter gritting and the like. The authors present two lower bounds and two heuristics for the CARP-IF. The first lower bound is based on the fact that the RPP is a special case of the CARP-IF, and therefore uses a relatively tight RPP lower bound based on Branch-and-Cut to bound the CARP-IF. The second lower bound is a relaxation lower bound of an ILP formulation based on dead-heading variables. The first heuristic they present for the problem is based on constructing an RPP-tour and splitting the tour into appropriate portions while connecting to the intermediate facilities. The second heuristic is based on solving the classical CARP in a modified network, transforming the solution to a CARP-IF solution in the original network and making some adjustments to restore feasibility.

\subsection{CARP with Mobile Depots}

Filippi and Del Pia, [46], consider a version of the CARP with two different types of servicing vehicles, where only one of them unloads at the depot. The other type of vehicle unloads onto the first type. With this setup, besides the routing of each type of vehicles, it must be decided at what time two vehicles must meet at some node in order to perform this unload action. This problem is encountered in a real life refuse collection problem, where satellite vehicles with small capacity unload into one of several large vehicles, which in turn are the only ones to unload at the depot. The authors solve the problem 
with a modified version of the Variable Neighborhood Descent algorithm first presented by Hertz and Mittaz, [64].

The CARP with Refill Points (CARP-RP) could be considered to be a Multi Depot CARP where the depots are traveling to the vehicles to refill, [5]. In this problem, two types of vehicles are given. The usual service vehicles that service the edges by traversing them, and the refilling vehicles that can meet the service vehicle at any point in the graph for refilling purposes. A mathematical model is given for the CARP-RP and a Cutting Plane algorithm for solving the problem is suggested by Amaya in [5].

\subsection{CARP with Vehicle/Site Dependencies}

The CARP with Vehicle/Site Dependencies, studied by Sniezek in [87], is a variation of the CARP with several types of vehicles. The problem is defined such that not all edges can be serviced or traversed by all types of vehicles. Ball et al., [14], suggest a vehicle decomposition algorithm for solving an instance of this problem which they encountered in a refuse collection application. In [24], Bodin and Sniezek propose a solution procedure for the problem, which is based on a composite approach consisting of an Initial Fleet Mix Generator, a Mathematical Programming Procedure, and a Measure of Goodness function.

\subsection{Periodic CARP}

The Periodic CARP (PCARP) is defined as the CARP where a long time horizon is considered such that each demand edge requires service more than once. This situation sometimes occurs in refuse collection where each household is serviced two or three times a week on a rolling schedule. Here, it must be taken into account that the problem may require a minimum and maximum number of days between each service of the same street. A mathematical formulation of the problem is given by Chu et al. in [33], where three heuristics are also suggested for obtaining feasible solutions. Lacomme et al., [73], suggests a Generic Algorithm for solving the problem. This algorithm is an extension of the algorithm presented by the same authors in [71]. A Scatter Search algorithm for PCARP is suggested by Chu et al., [34], and two lower bounds are given by the same authors in [32]. Both of these are based on lower bounds for the classical CARP defined on a transformed graph.

\subsection{Stochastic CARP}

The Stochastic CARP (SCARP), first suggested by Fleury et al., [48, 50], is identical to the classical CARP except that the demand on the edges is a random variable. This problem occurs in practice in Refuse Collection, Mail Delivery, and Snow Removal where the exact demand is not known. In [49], Fleury et al. study the quality of solutions for the SCARP when the solutions are obtained with algorithms for the classical deterministic CARP. 
They explore how the robustness of the solutions change when the deterministic problem is solved with a slightly smaller vehicle capacity. In [47], Fleury et al. present a Memetic Algorithm for the SCARP, which is an extension of the algorithm suggested by Lacomme et al. in [74]. The results obtained are compared to the results generated by algorithms for the classical CARP based on the average demand.

\section{The Next Decade of Capacitated Arc Routing}

In the preceding two sections, we have given an overview of the latest research on the Capacitated Arc Routing Problem and its extensions. In this section, we will offer our directions for future research within the area.

In general, there are two directions for future research. The first one goes toward size, speed, and quality, and the second one goes toward flexibility. Even though the ultimate goal is to attain the two goals simultaneously, we will elaborate on them separately.

In other words, the goal in the first direction is the development of faster algorithms to obtain better results for larger problem instances. As we have pointed out, the past decade has seen many contributions within the area of metaheuristics. Several of these can be used to obtain solutions that are within a few percent of the optimum for the existing benchmark instances. The new challenge is to use huge instances so that it will be possible to identify the new, better contributions with respect to quality and speed. Few attempts have been made to solve the CARP exactly, where some are based on converting the problem into node routing. This has lead to the development of several classes of cuts and separation routines. To reach the goal of obtaining exact solutions of larger instances in shorter time, new cuts need to be explored and the construction of exact methods that exploit the network structure of the problem might be beneficial.

In the previous section we have seen that most of the real life applications of the CARP contain different kinds of additional constraints such as vehicles of various sizes, time windows, or several resource types. The direction of flexibility is closely linked to a goal of usefulness for such real life problems. Some of the research in the area has already dealt with this aspect - in particular from a heuristic point of view, but the area is still open for further exploration, in particular with respect to exact methods. Moreover, with a few exceptions, all published material on the CARP deals with a deterministic and static setup. It is well known that many real life arc routing problems are either stochastic or dynamically changing, and therefore we recommend that these issues be further explored. 


\section{Acknowledgement}

The author would like to thank anonymous referees for their suggestions for improving this chapter.

\section{References}

1. D. Ahr. Contributions to multiple postmen problems. PhD thesis, University of Heidelberg, 2004.

2. D. Ahr and G. Reinelt. A tabu search algorithm for the min-max $k$-chinese postman problem. Computers and Operations Research, 33(12):3403-3422, 2006.

3. M.T. Almeida and M.C. Mourão. Lower-bounding and heuristic methods for a refuse collection vehicle routing problem. European Journal of Operational Research, 121:420-434, 2000.

4. L. Amado and M.C. Mourão. Heuristic method for a mixed capacitated arc routing problem: A refuse collection application. European Journal of Operational Research, 160(1):139-153, 2005.

5. A. Amaya, A. Langevin, and M. Trépanier. The capacitated arc routing problem with refill points. Operations Research Letters, 35(1):45-53, 2007.

6. A. Amberg, W. Domschke, and S. Voß. Multiple center capacitated arc routing problems: A tabu search algorithm using capacitated trees. European Journal of Operational Research, 124:360-376, 2000.

7. A. Amberg and S. Voß. A hierarchical relaxations lower bound for the capacitated arc routing roblem. Proceedings of the 35th Annual Hawaii International Conference on System Sciences, 3, 2002.

8. S.K. Amponsah and S. Salhi. The investigation of a class of capacitated arc routing problems: The collection of garbage in developing countries. Waste Management, 24:711-721, 2004.

9. J. Aráoz, E. Fernández, and C. Zoltan. Privatized rural postman problem. Computers and Operations Research, 33(12):3432-3449, 2006.

10. A.A. Assad and B.L. Golden. Arc routing methods and applications, in Handbooks in operations research and management science, Volume 8 - Network routing, pages 375-483. Michael O. Ball and T.L. Magnanti and C.L. Monma and George L. Nemhauser, 1995.

11. A.A. Assad, B.L. Golden, and W.L. Pearn. Transforming arc routing into node routing problems. Computers and Operations Research, 14(4):285-288, 1987.

12. E.K. Baker, J.S. DeArmon, and B.L. Golden. Computational experiments with algorithms for a class of routing problems. Computers and Operations Research, 10(1):47-59, 1983.

13. R. Baldacci and V. Maniezzo. Exact methods based on node routing formulations for undirected arc routing problems. Networks, 47(1):52-60, 2006.

14. M. Ball, L. Bodin, L. Levy, and J. Sniezek. Capacitated arc routing problem with vehicle-site dependencies: The Philadelphia experience, in The vehicle routing problem. Paolo Toth and Daniele Vigo, 2001.

15. J.M. Belenguer, E. Benavent, P. Lacomme, and C. Prins. Lower and upper bounds for the mixed capacitated arc routing problem. Computers and Operations Research, 33(12):3363-3383, 2006. 
16. J.M. Belenguer. http://www.uv.es/ ${ }^{\sim}$ belengue/carp.html.

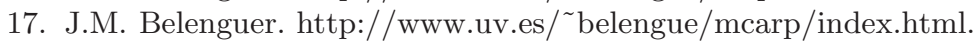

18. J.M. Belenguer and E. Benavent. The capacitated arc routing problem: Valid inequalities and facets. Computational Optimization and Applications, 10:165$187,1998$.

19. J.M. Belenguer and E. Benavent. A cutting plane algorithm for the capacitated arc routing problem. Computers and Operations Research, 30(5):705-728, 2003.

20. J.M. Belenguer, E. Benavent, and D. Gómez-Cabrero. Cutting plane and column generation for the capacitated arc routing problem. ORP3 Meeting, Valencia, 2005 .

21. E. Benavent, A. Corberán, and J.M. Sanchis. Linear programming based methods for solving arc routing problems, in Arc routing - Theory, solutions and applications. M. Dror, editor. Kluwer Academic Publishers, 2000.

22. E. Benavent, V. Campos, A. Corberán, and E. Mota. The capacitated arc routing problem: Lower bounds. Networks, 22:669-690, 1992.

23. P. Beullens, D. Cattrysse, L. Muyldermans, and D. Van Oudheusden. A guided local search heuristic for the capacitated arc routing problem. European Journal of Operational Research, 147:629-643, 2003.

24. L. Bodin and J. Sniezek. Using mixed integer programming for solving the capacitated arc routing problem with vehicle/site dependencies with an application to the routing of residential sanitation collection vehicles. Annals of Operations Research, 144(1):33-58, 2006.

25. L.D. Bodin and S.J. Kursh. A computer-assisted system for the routing and scheduling of street sweepers. Operations Research, 26(4):525-537, 1987.

26. J. Brandâo and R.W. Eglese. A deterministic tabu search algorithm for the capacitated arc routing problem (CARP). Computers and Operations Research, to appear.

27. J.F. Campbell, A. Langevin, and N. Perrier. A survey of models and algorithms for winter road maintenance. Part III: Vehicle routing and depot location for spreading. Computers and Operations Research, 34(1):211-257, 2007.

28. J.F. Campbell, A. Langevin, and N. Perrier. A survey of models and algorithms for winter road maintenance. Part IV: Vehicle routing and fleet sizing for plowing and snow disposal. Computers and Operations Research, 34(1):258-294, 2007.

29. D. Cattrysse, T. Lotan, L. Muyldermans, and D. Van Oudheusden. Districting for salt spreading operations. European Journal of Operational Research, 139:521-532, 2002.

30. D. Cattrysse, L. Muyldermans, and D. Van Oudheusden. District design for arcrouting applications. Journal of the Operational Research Society, 54:1209-1221, 2003.

31. N. Christofides. The optimum traversal of a graph. OMEGA, The International Journal of Management Science, 1(6):719-732, 1973.

32. F. Chu, N. Labadi, and C. Prins. Lower bounds for the periodic capacitated arc routing problem The Odysseus 2003 conference, 2003.

33. F. Chu, N. Labadi, and C. Prins. Heuristics for the periodic capacitated arc routing problem. Journal of Intelligent Manufacturing, 16:243-251, 2005.

34. F. Chu, N. Labadi, and C. Prins. A scatter search for the periodic capacitated arc routing problem. European Journal of Operational Research, 169(2):586-605, 2006 . 
35. M. P de Aragão, H. Longo, and E. Uchoa. Solving capacitated arc routing problem using a transformation to the CVRP. Computers and Operations Research, 33(6):1823-1837, 2006.

36. K.F. Doerner, R.F. Hartl, V. Maniezzo, and M. Reimann. An ant system metaheuristic for the capacitated arc routing problem. Preprints of 5th Metaheuristics International Conference, Kyoto, 2003.

37. M. Dror, editor. Arc routing - Theory, solutions and applications. Kluwer Academic Publishers, 2000.

38. M. Dror, H.I. Stern, and P. Trudeau. Postman tour on a graph with precedence relation on arcs. Networks, 17:283-294, 1987.

39. J. Edmonds and E.L. Johnson. Matching, euler tours and the chinese postman. Mathematical Programming, 5(1):88-124, 1973.

40. R.W. Eglese. Routing winter gritting vehicle. Discrete Applied Mathematics, 48:231-244, 1994.

41. R.W. Eglese and A.N. Letchford. The rural postman problem with deadline classes. European Journal of Operational Research, 105:390-400, 1998.

42. R.W. Eglese and A.N. Letchford. Polyhedral theory for arc routing problems, in Arc routing - Theory, solutions and applications. M. Dror, editor. Kluwer Academic Publishers, 2000.

43. R.W. Eglese and L.Y.O. Li. An interactive algorithm for vehicle routeing for winter-gritting. Journal of the Operational Research Society, 47:pp. 217-228, 1996.

44. H.A. Eiselt, M. Gendreau, and G. Laporte. Arc routing problems, Part 1: The chinese postman problem. Operations Research, 43(2):231-242, 1995.

45. H.A. Eiselt, M. Gendreau, and G. Laporte. Arc routing problems, Part 2: The rural postman problem. Operations Research, 43(3):399-414, 1995.

46. C. Filippi and A. Del Pia. A variable neighborhood descent algorithm for a real waste collection problem with mobile depots. International Transactions in Operational Research, 13:125-141, 2006.

47. G. Fleury, P. Lacomme, and C. Prins. Evolutionary algorithms for stochastic arc routing problems. Lecture Notes in Computer Science, 3005:501-512, 2004.

48. G. Fleury, P. Lacomme, and C. Prins. Stochastic capacitated arc routing problems. Research Report LIMOS/RR-05-12, 2005.

49. G. Fleury, P. Lacomme, C. Prins, and W. Ramdana-Chérif. Robustness evaluation of solutions for the capacitated arc routing problem. Conference, AI Simulation and Planning in High Autonomy Systems. ISBN 1-56555-242-3, pages 290-295, 2002.

50. G. Fleury, P. Lacomme, C. Prins, and W. Ramdane-Cherif. Improving robustness of solutions to arc routing problems. Journal of the Operational Research Society, 56:526-538, 2005.

51. M. Fleury. Deux problemes de geometrie de situation. Journal de Mathematiques Elementaires, pages 257-261, 1883.

52. G.N. Frederickson. Approximation algorithms for some postman problems. Journal of Association fot Computing Machinery, 26(3):538-554, 1979.

53. G.N. Frederickson, M.S. Hecht, and C.E. Kim. Approximation algorithms for some routing problems. SIAM Journal of Computing, 7(2):178-193, 1978.

54. M. Gendreau, J.Y. Potvin, and M. Tagmouti. Arc routing problems with timedependent service costs. European Journal of Operational Research, 181(1):3039, 2007. 
55. G. Ghiani, F. Guerriero, G. Improta, and R. Musmanno. Waste collection in southern Italy: Solution of a real-life arc routing problem. International Transactions in Operational Research, 12:135-144, 2005.

56. G. Ghiani, G. Improta, and G. Laporte. The capacitated arc routing problem with intermediate facilities. Networks, 37(3):134-143, 2001.

57. F.W. Glover and G.A. Kochenberger, editors. Handbook of metaheuristics. Kluwer Academic Publishers, 2003.

58. B.L. Golden and R.T. Wong. Capacitated arc routing problems. Networks, 11:305-315, 1981.

59. P. Greistorfer. A tabu scatter search metaheuristic for the arc routing problem. Computers and Industrial Engineering, 44(2):249-266, 2003.

60. P. Haastrup, V. Maniezzo, M. Mattarelli, F. Mazzeo Rinaldi, I. Mendes, and M. Paruccini. A decision support system for urban waste management. European Journal of Operational Research, 109:330-341, 1998.

61. A. Hertz. Recent Trends in Arc Routing. in Graph theory, Combinatorics and algorithms: Operations research/computer science interfaces series, M.C. Golumbic and I.B.A Hartman. 2005.

62. A. Hertz, G. Laporte, and M. Mittaz. A tabu search heuristic for the capacitated arc routing problem. Operations Research, 48(1):129-135, 2000.

63. A. Hertz, G. Laporte, and P. Nanchen-Hugo. Improvement procedures for the undirected rural postman problem. INFORMS Journal of Computing, 11(1):53$62,1999$.

64. A. Hertz and M. Mittaz. A variable neighborhood descent algorithm for the undirected capacitated arc routing problem. Transportation Science, 35(4):425434, 2001.

65. R. Hirabayashi, N. Nishida, and Y. Saruwatari. Node duplication lower bounds for the capacitated arc routing problems. Journal of the Operations Research Society of Japan, 35(2):119-133, 1992.

66. R. Hirabayashi, N. Nishida, and Y. Saruwatari. Tour construction algorithm for the capacitated arc routing problem. Asia-Pacific Journal of Operational Research, 9:155-175, 1992.

67. K. Jansen. An approximation algorithm for the general routing problem. Information Processing Letters, 41:333-339, 1992.

68. A.H.G. Rinnooy Kan and J.K. Lenstra. On general routing problems. Networks, 6:273-280, 1976.

69. M. Kiuchi, R. Hirabayashi, Y. Saruwatari, and Y. Shinano. An exact algorithm for the capacitated arc routing problem using parallel branch and bound method. Abstracts of the 1995 Spring National Conference of the Oper. Res. Soc. of Japan, pages pp. 28-29, 1995.

70. N. Labadi, C. Prins, and M. Reghioui. GRASP with path relinking for the capacitated arc routing problem with time windows. Lecture Notes in Computer Science, 4448:722-731, 2007.

71. P. Lacomme, C. Prins, and W. Ramdana-Chérif. Competitive genetic algorithms for the capacitated arc routing problem and its extensions. Lecture Notes in Computer Science, 2037:473-483, 2001.

72. Philippe Lacomme, Christian Prins, and Wahiba Ramdana-Chérif. An integer linear model for general routing problems. Technical Report., 2003.

73. Philippe Lacomme, Christian Prins, and Wahiba Ramdana-Chérif. Evolutionary algorithms for periodic arc routing problems. European Journal of Operational Research, 165:535-553, 2005. 
74. Philippe Lacomme, Christian Prins, and Wahiba Ramdane-Cherif. Competitive memetic algorithms for arc routing problems. Annals of Operations Research, 131:159-185, 2004.

75. Philippe Lacomme, Christian Prins, and Marc Sevaux. Multiobjective capacitated arc routing problem. Lecture Notes in computer Science, 2632:550-564, 2003.

76. Philippe Lacomme, Christian Prins, and Marc Sevaux. A genetic algorithm for a bi-objective capacitated arc routing problem. Computers and Operations Research, 33(12):3473-3493, 2006.

77. Philippe Lacomme, Christian Prins, and Alain Tanguy. First competitive ant colony scheme for the CARP. Lecture Notes in Computer Science, 3172:426-427, 2004.

78. André Langevin, Fabien Marzolf, and Marting Trépanier. Road network monitoring: Algorithms and a case study. Computers and Operations Research, 33(12):3494-3507, 2006.

79. Adam N. Letchford and Amar Oukil. Exploiting sparsity in pricing routines for the capacitated arc routing problem. Working paper, Department of Management Science, Lancaster University, 2006.

80. Arne Løkketangen and Johan Oppen. Arc routing in a node routing environment. Computers and Operations Research, 33(4):1033-1055, 2006.

81. Renata Mansini and M. Grazia Speranza. A linear programming model for the separate refuse collection service. Computers and Operations Research, 25(7/8):659-673, 1998.

82. Kwan Mei-Ko. Graphic programming using odd or even points. Chinese Mathematics, 1:237-277, 1962.

83. E. Minieka. The chinese postman problem for mixed networks. Management Science, 25:643-648, 1979.

84. C.S. Orloff. A fundamental problem in vehicle routing. Networks, 4:35-64, 1974.

85. Christos H. Papadimitriou. On the complexity of edge traversing. Journal of the Association for Computing Machinery, 23(3), 1976.

86. Horst Sachs, Michael Stiebitz, and Robin J. Wilson. An historical note: Euler's Königberg letters. Journal of Graph Theory, 12(1):133-139, 1988.

87. John Sniezek. The capacitated arc routing problem with vehicle/site dependencies: An application of arc routing and partitioning. PhD thesis, University of Maryland, 2001.

88. Gunduz Ulusoy. The fleet size and mix problem for capaciteted arc routing. European Journal of Operational Research, 22:329-337, 1985.

89. Scott Allen Welz. Optimal solutions for the capacitated arc routing problem using integer programming. PhD thesis, University of Cincinnati, 1994.

90. Zaw Win. Contributions to routing problems. PhD thesis, University of Augsburg, 1988.

91. Sanne Wøhlk. http://www.wohlk-son.dk/sanne/research_carptw.html.

92. Sanne Wøhlk. Contributins to arc routing. PhD thesis, University of Southern Denmark, 2005.

93. Sanne Wøhlk. New lower bound for the capacitated arc routing problem. Computers and Operations Research, 33(12):3458-3472, 2006. 